\title{
Photography Transillumination Techniques: Multicystic Peritoneal Mesothelioma
}

\author{
Marie Jones and Faheez Mohamed
}

Medical photography can be both challenging and rewarding, particularly when faced with less than ideal situations and under pressure of time, environment, and clinicians' expectations. Clinical photographs are a scientific record, and therefore should be accurately focused, exposed and framed. They should fulfil the brief and illustrate the subject effectively. I will describe a photography technique that has been invaluable in the documentation and diagnosis of a rare clinical condition, (multicystic peritoneal mesothelioma) and in informing fellow clinicians of the visual appearance of this disease.

\section{OPEN ACCESS}

\section{Keywords}

Multicystic peritoneal mesothelioma, medical photography, medical imaging, transillumination lighting technique, intraoperative, colorectal disease, cytoreductive surgery (CRS), hyperthermic intraperitoneal chemotherapy (HIPEC), peritoneal malignancy

\section{Introduction - the clinical condition}

Multicystic peritoneal mesothelioma is a rare condition that commonly presents with abdominal pain, tenderness, and distension. It is often discovered incidentally in women undergoing investigation for infertility, and the diagnosis can be challenging due to the rarity of the condition, as well as the non-specific nature of symptoms. It predominantly affects, women of reproductive age. It is typically a slowly progressive condition that rarely spreads outside of the abdomen (Chua et al. 2011).

\section{Multicystic Peritoneal Mesothelioma}

Although the peritoneum is the most common tissue of origin, multicystic mesothelioma can also originate on other serosal membranes (pleura, spermatic cord, tunica vaginalis, and pericardium). Studies vary as to the proportion of patients with a history of previous surgery, pelvic inflammatory disease, or endometriosis, suggesting that chronic peritoneal irritation could be a precipitating factor.

Imaging with ultrasound (US) (Figure 1a) and Computed Tomography (CT), Magnetic Resonance Imaging (MRI)
(Figure 1b) can be useful with characteristic appearances in these modalities.

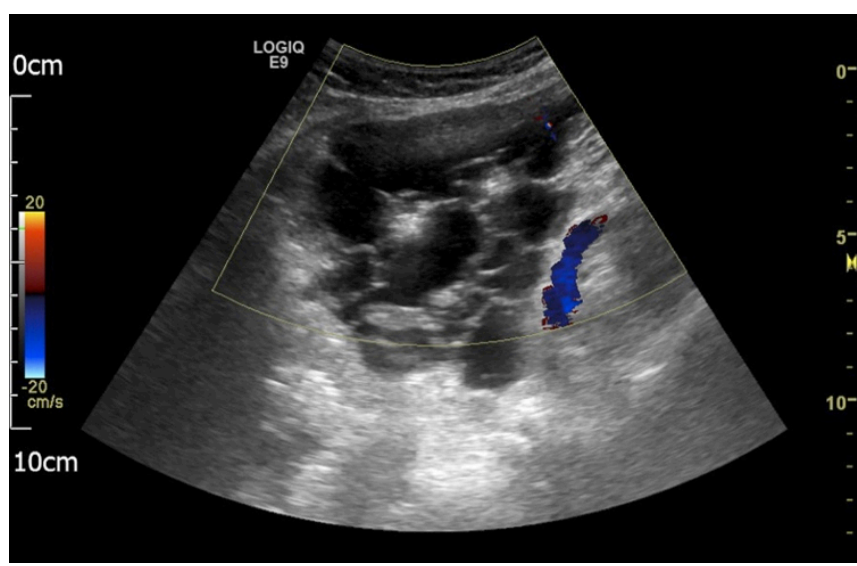

Figure 1a. Initial Ultrasound (US) of the abdomen.

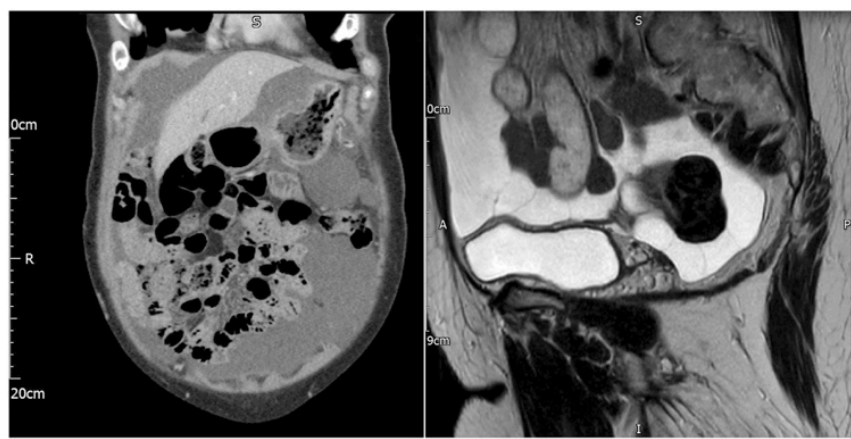

Figure 1b. Computed Tomography (CT) and Magnetic Resonance Imaging (MRI) of the abdomen.

In women, cysts often adhere closely to the uterus and the ovaries. An ultrasound usually shows anechoic to mildly echogenic, multiseptated cystic structures in the pelvis with a varying number of lobulations and cysts.

On rare occasions, intra-abdominal fluid or hemorrhage is also reported. On CT, multi-cystic peritoneal mesothelioma usually appears as low-density, multi-loculated, multi-cystic, thin walled lesions that may engulf the surrounding soft tissue; however, invasion has not been reported. On MRI, they appear as multi-loculated cystic masses that are hypointense on T1 weighted images and hyper to intermediate intensity on T2 weighted sequences. The walls may demonstrate mild enhancement with contrast. Fat within the cysts has not been demonstrated. 
No standard treatment exists for this condition. Due to the high rate of recurrence following simple surgical excision and risk of malignant transformation, an aggressive approach has been proposed with cytoreductive surgery combined with Hyperthermic Intraperitoneal Chemotherapy (HIPEC).

Postoperatively patients sometimes need CT scans to look for intra-abdominal collections that may benefit from drainage, as well as routine ultrasound scans of the leg veins to look for blood clots. The average length of stay in hospital after surgery is 3 weeks. After discharge, patients undergo annual surveillance CT scans with tumor marker blood tests (CEA, CA 125, CA 19-9) (Gilani et al. 2018).

This patient underwent cytoreductive surgery with HIPEC successfully in Basingstoke and is currently under surveillance with annual CT scans and tumor markers (Figure 1c), the patient also had an X-ray at this stage. (Figure 1d)

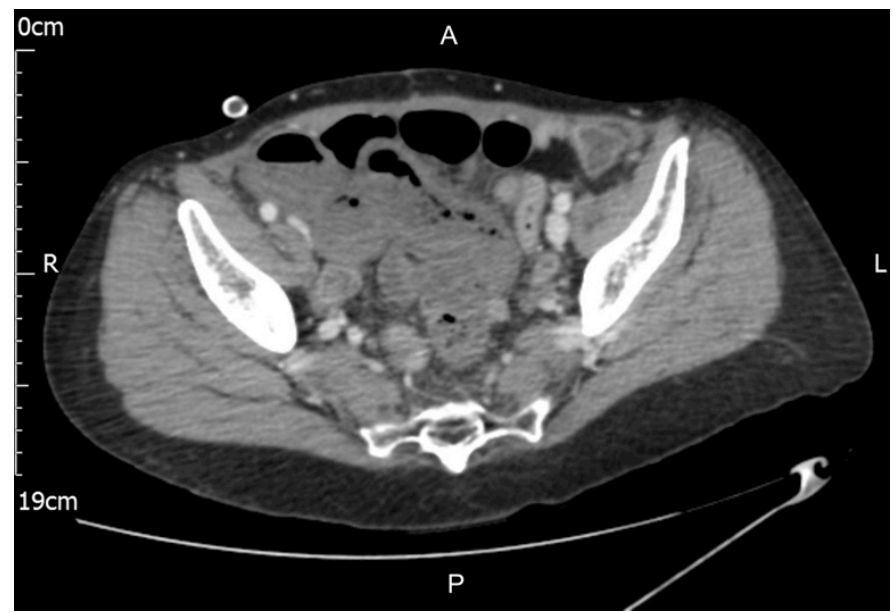

Figure 1c. CT of abdomen 10 months post-operative for surveillance.

Scans are performed annually for 6 years then at $8,11,15$ and 20 years after surgery. If a complete tumor removal can be achieved, there is a good chance of cure. Most patients return to pre-illness quality of life at 12 months from surgery. Outcomes are good after complete tumor removal with 5-year survival of around $80 \%$.

\section{The Photography Request}

Thursday morning - the phone rang, "Can you come to Theatre 2 straight away please? We have a rare condition to be photographed." The usual scenario for a medical photographer. Arriving a few minutes later in the operating theatre I was welcomed by the surgeon (second author) with, "I need really good photos of this please."

The patient's abdominal cavity was open, and the contents were spilled out onto the sterile operating drapes. (Figure 2a)

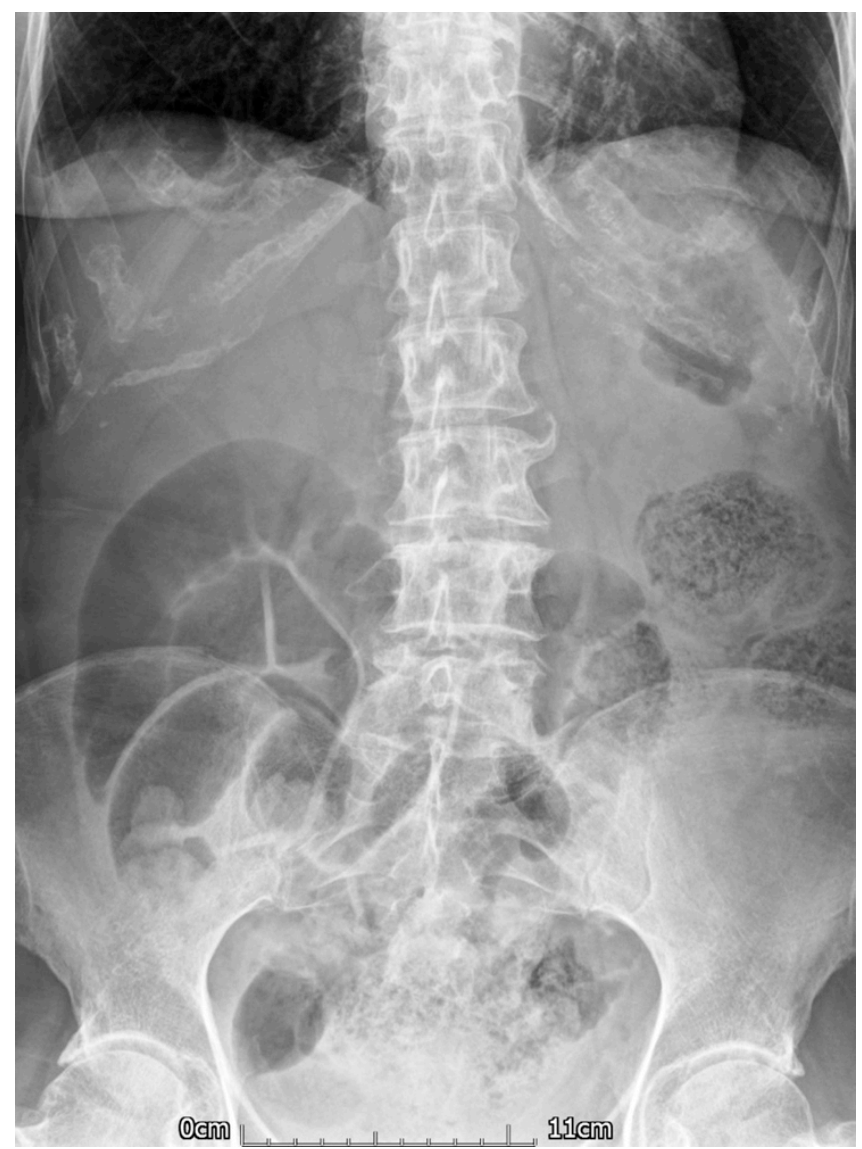

Figure 1d. X-ray 10 months post-operative for surveillance.

I could see that I needed to document the abdominal anatomy and tumors and depict the frailty of the specimen's membrane and the fluid it contained. The surgeons' hands in the images give some idea as to the size of the anatomy. The surgeon stated that the images were needed as part of the clinical diagnostic process, as well as being used in scientific research and teaching. I knew immediately that there was also 'artistic' potential with this subject - I was up to the challenge.

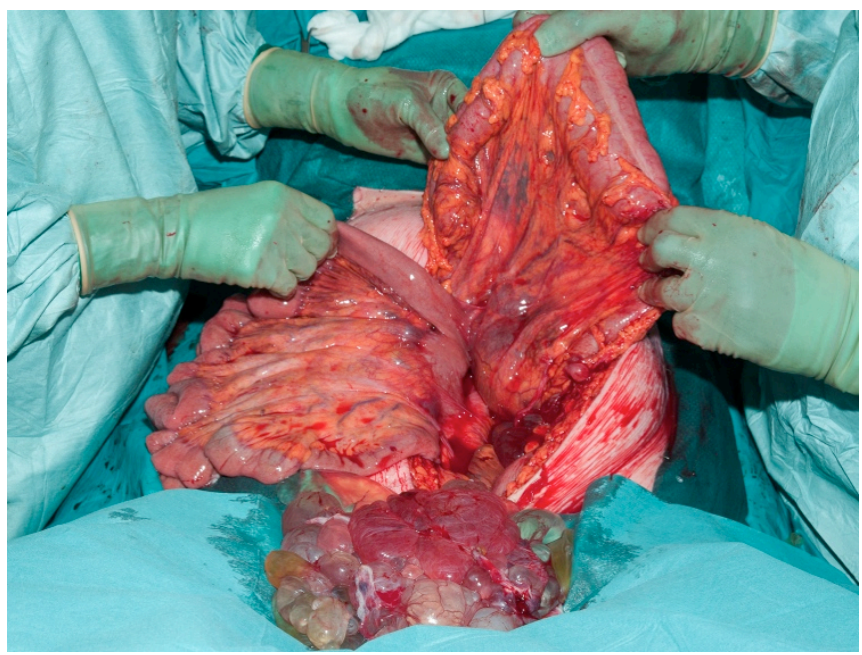

Figure 2a. Photograph taken using hand-held flash. 


\section{Technical Challenges}

\section{First Challenge: Speed}

The specimen (one of several) had just been removed by the surgeon from the patient's abdomen, and the surgeon was in the process of removing further tumors. (Figure 2b) In order to prevent the patient being under anesthesia any longer than necessary, the surgeon needed me to take all photographs as quickly as possible, so that he could continue with the operation.

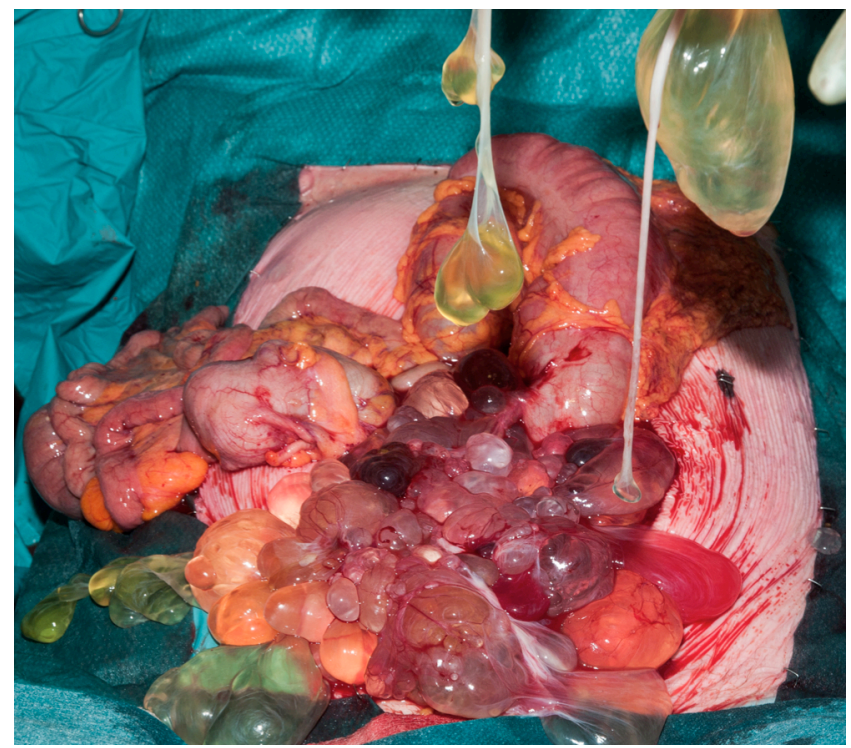

Figure 2b. Abdominal cavity exposing tumors in situ and in the process of extraction.

\section{Second Challenge: Lighting using the hand-held flash} whilst balancing ISO, shutter speed, and aperture

The equipment and settings that I used with this patient were: crop sensor DSLR with $60 \mathrm{~mm}$ macro lens; Speedlight handheld flashgun with extension synch cable; ISO 200 for an acceptable level of grain/ noise; $1 / 160^{\text {th }} \mathrm{sec}$ (to synchronize with the flash) aperture at $\mathrm{f} / 16$. The flash was directed towards and bounced off the operating lights, (Figure 2c); this method allowed me individual flexibility with the lighting for each image. I rarely use a ringflash (except for lighting a body cavity) due to the rather 'flat' appearance that this type of lighting gives.

With some of the specimens, the use of flash lighting did not fully demonstrate the fragile nature of the membranes or the fluid contained within; therefore, I needed to improvise the lighting technique.

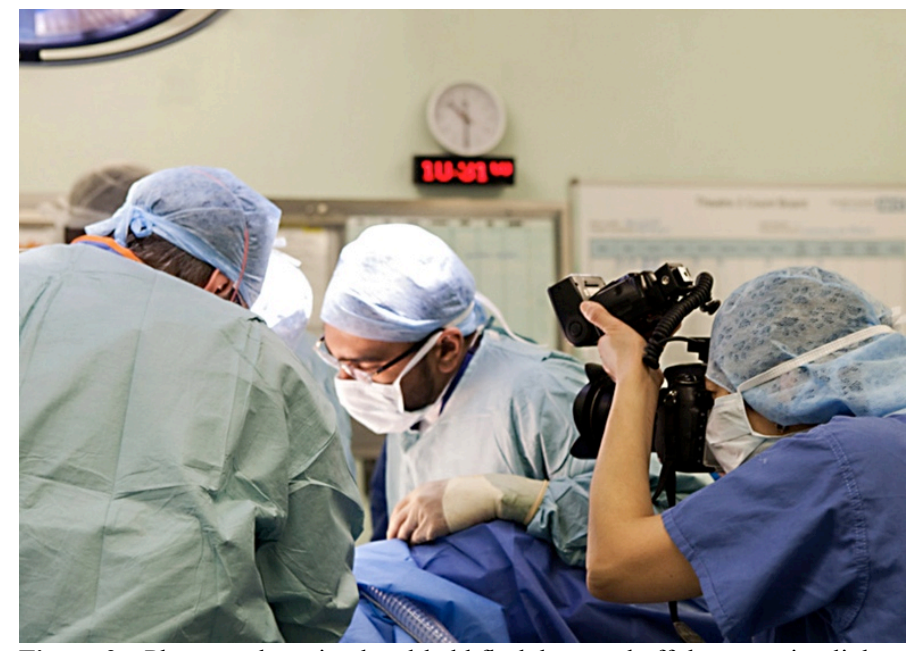

Figure 2c. Photography using hand-held flash bounced off the operating lights above.

\section{Third Challenge: Lighting using the transillumination technique}

In order to demonstrate the delicate membranes and the color of the fluid within the specimen, I then applied the transillumination technique as defined by Kaliyadan et. al., (2018) and Ray (1999, 86-87) respectively:

"Transillumination in medicine refers to tissue visualization after transmitting light through it."

"The interior detail of a subject with a translucent covering or outer structure can be made visible using selective absorption by placing a light source behind it to provide transillumination."

The surgeon extended the membrane of the specimen to demonstrate its length, texture and fragility. I asked him to hold the specimen forward of his body so that the operating lights could be re-positioned behind the specimen to transilluminate the fluid whilst ensuring that the lights did not contaminate the operative field. The surgeon's sterile gown provided a suitable uniform background, so drawing the viewer's eye to the capsules of the lit specimen. (Figure 2d)

Using a tripod to allow a slower shutter speed would have been beneficial; however, due to limited space and infection control policies in the operating theatre a tripod could not be used. Therefore, the ISO was increased to 1600 to allow for an exposure of $1 / 160^{\text {th }} / \mathrm{sec}$ at $\mathrm{f} / 11$. A reasonable aperture was required to ensure that as much of the specimen as possible was imaged sharply, even when not in the main plane of focus.

Each of the operating theatres have different makes and models of room lighting and operating lighting used over the surgical field; therefore, the color temperature varies from one theatre to another. When using flash lighting I request that the surgical lights are turned off where possible (or away from the subject) in order to eliminate an illumination 'hot-spot' and color 
temperature imbalance to that of the flashgun (Nikon Speedlight).

With this subject, I did not alter the white balance, as there was no quick or easy method to allow for color temperature change due to now using the operating lights as the key lighting instead of the flashgun. Figure 2e demonstrates a comparison of an image taken with hand-held flash (left) together with an image taken using the transillumination technique (right).

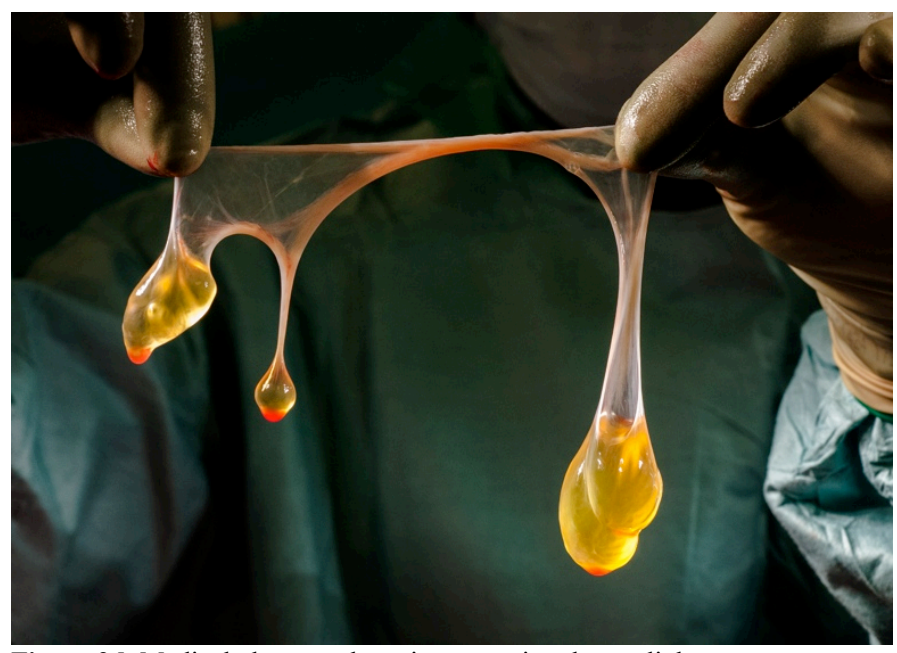

Figure 2d. Medical photography using operating theatre lights to transilluminate the specimen.
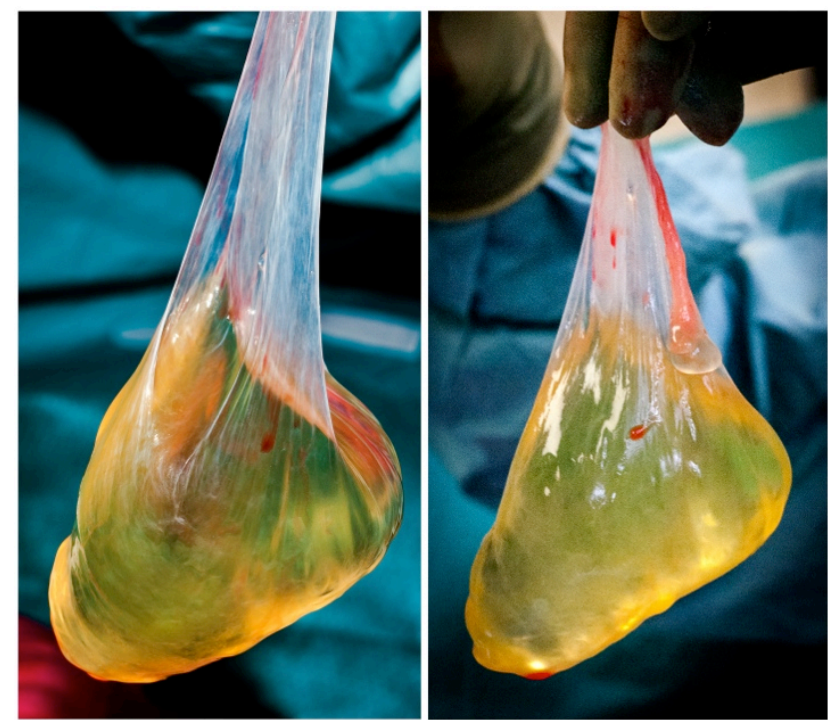

Figure 2e. Image (left) taken using hand-held flash and image (right) taken using operating theatre lights.

Many images were taken, using the hand-held flash and using the transillumination technique. Once I had documented all of the abdominal organs and tumors, the operating lights were returned to the more usual position above the patient to illuminate the abdominal cavity so that the surgical procedure could continue. All photographs were taken in RAW format and processed with Adobe Camera RAW.

\section{Use of Images}

The full set of 20 clinical photographs were included in the patient's electronic medical record; a few of these are included here in Figures 2a, 2b, 2d, 2e, 3a, 3b.

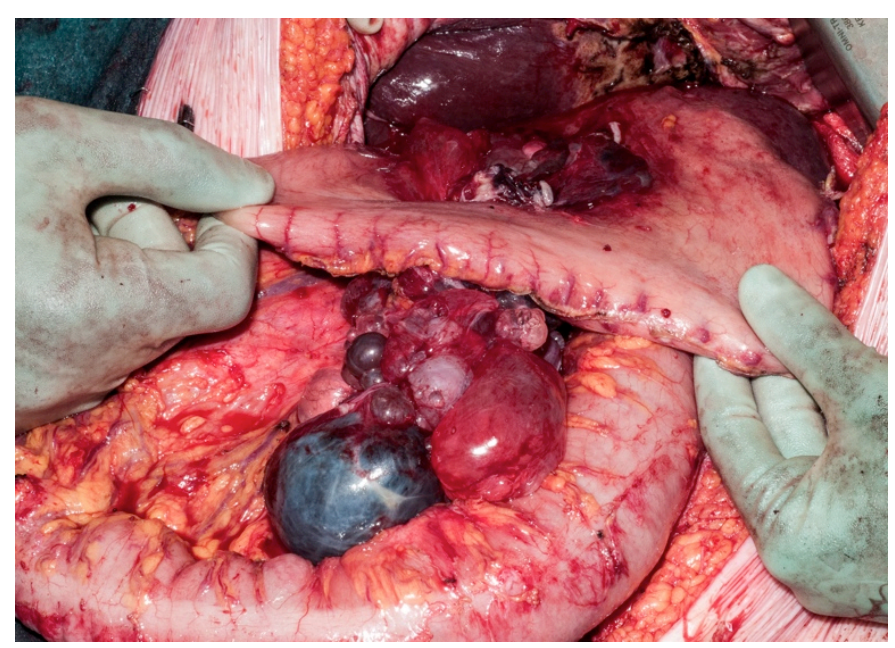

Figure 3a. Abdomen to show position of organs and tumors.

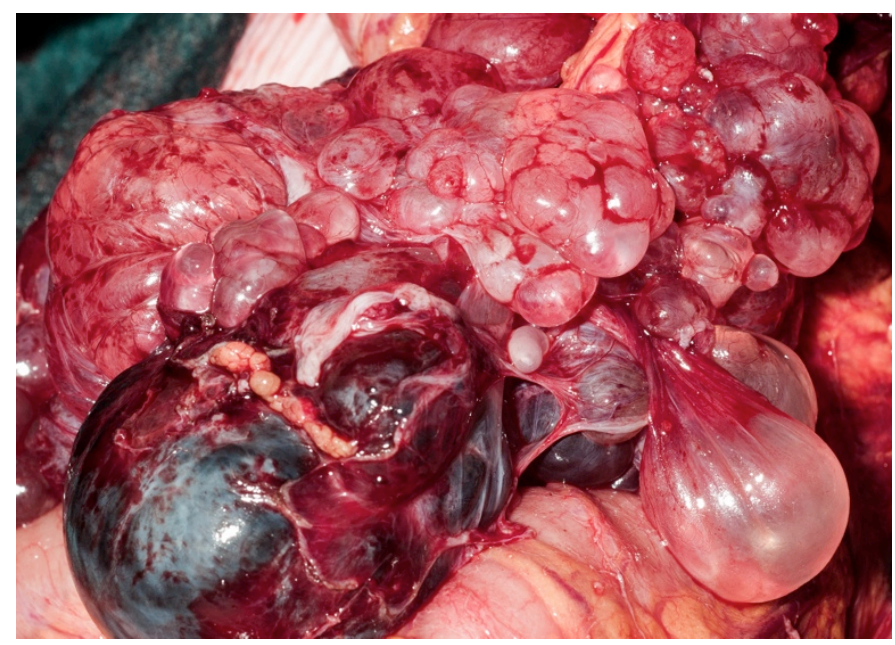

Figure 3b. Abdominal organs and tumors.

The images were presented, together with radiographic imaging, (Figure 3c), at the Multidisciplinary Team Meeting (MDT) to enable the team to discuss the patient's on-going treatment following the initial surgery and chemotherapy. 


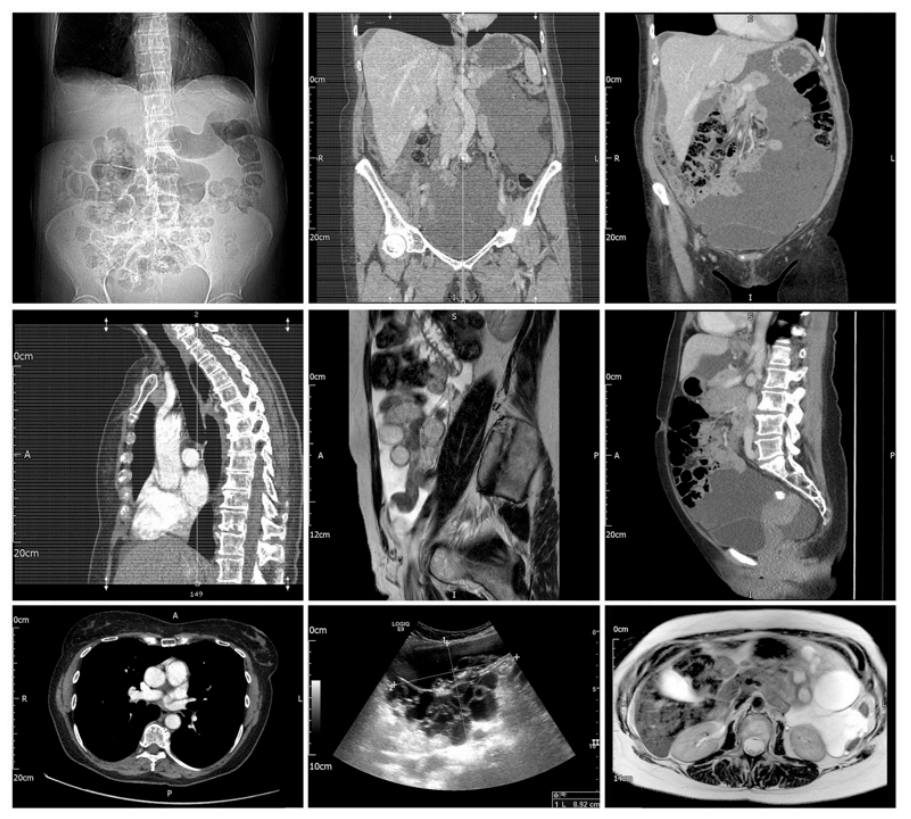

Figure 3c. Pre-operative computed tomography (CT) Sagittal aspect of abdomen and Ultrasound (US) of abdomen for discussion at MDT meeting.

\section{Value of Medical Photography}

This case study was used in research and the imaging was used to demonstrate to clinicians, the visual appearance of the disease to enable recognition and diagnosis of this rare condition.

The surgeon used these examples to encourage his peers to make use of the medical photography departments in their hospitals and allow the photographer a little extra time to obtain relevant, clinically valuable professional quality photographs.

The surgeon's opinion:

"In the management of rare diseases high quality medical photography is invaluable both to characterize uncommon conditions but also to raise awareness amongst clinicians and aid more rapid diagnosis.

Having an experienced medical photographer on site allows the surgeon to capture images in the most effective and striking way. It also provides the opportunity to describe the variety of presentations that one condition may have. When combined with other imaging modalities a compelling story can be told that effectively informs clinical practice.

Patients also find medical photographs fascinating, and being able to visualise their condition can be useful in helping them come to terms with their illness."

\section{Comparison of Images}

Additional examples of medical photographs demonstrating applications and advantages of the transillumination technique are described.

Pseudomyxoma peritonei (rare abdominal cancer): Figure 4a using hand-held flash lighting (intra-operative) demonstrates the tumors as fairly solid structures with some fluid areas; however, the use of transillumination demonstrates that the tumors are, in fact, entirely fluid-filled (Figure 4b); this finding is supported by Erne et.al., (2011): “...cystic lesions transilluminate, while solid tumors do not."

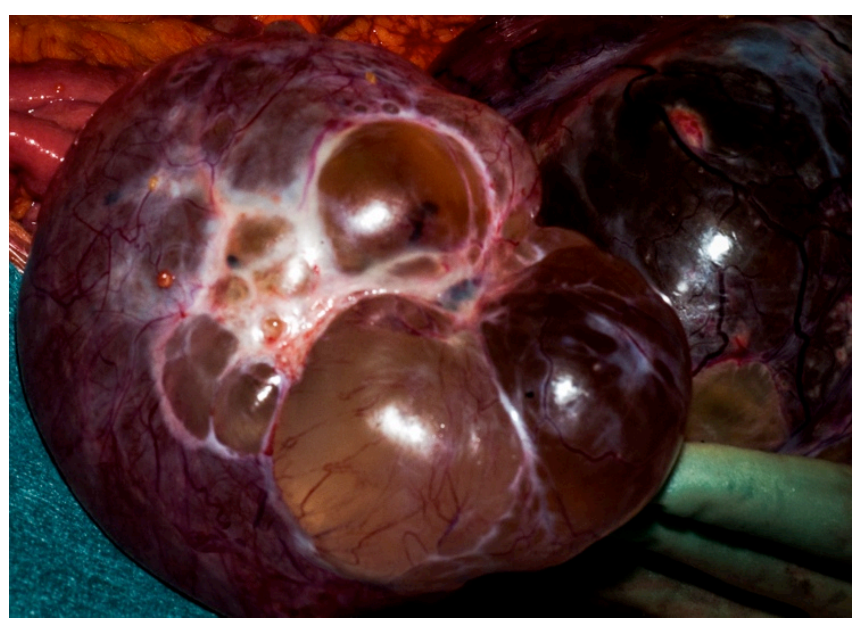

Figure 4a. Pseudomyxoma tumor using hand-held flash.

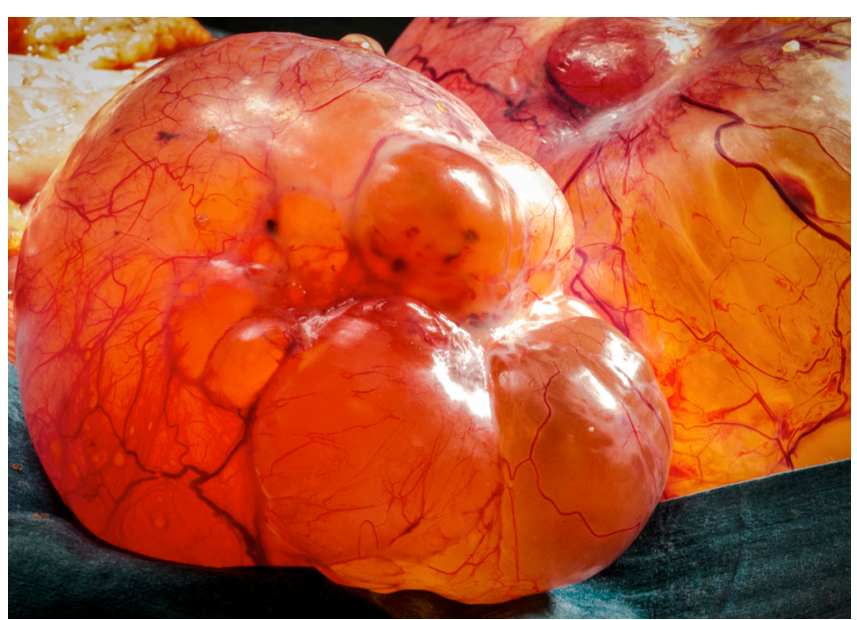

Figure 4b. Pseudomyxoma tumor using operating theatre lights to transilluminate. 


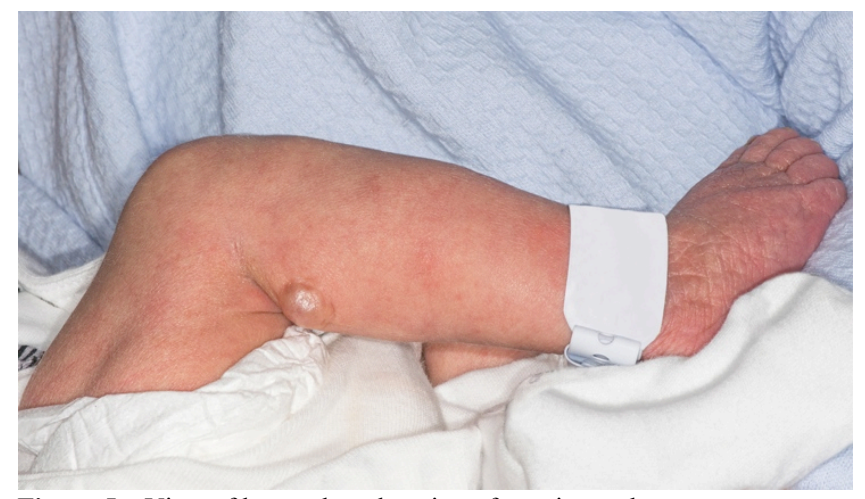

Figure 5a. View of leg to show location of cyst in newborn.

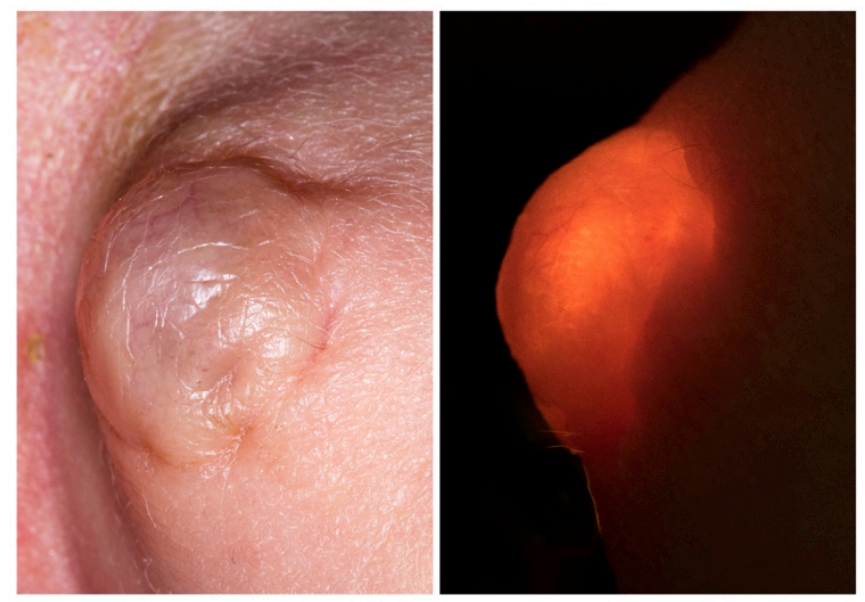

Figure 5b. Photographs taken with hand-held flash (left) compared with transilluminated cyst (right) in newborn.

Cyst in newborn: with a young baby it is preferable to use noninvasive means of imaging for diagnosis (Goren et. Al., 2001). The baby was photographed on the ward, but these techniques could equally be applied to photography in the clinic or photography studio. Figure 5a demonstrates the location of the cyst on the leg in a newborn. Figure 5b (left image), taken with hand-held flash demonstrates the surface anatomy of the cyst compared with the image on the right where a torch held below the leg was used to transilluminate the cyst to demonstrate the internal structure: the margins of soft tissue and fluid areas are clearly visible using transillumination. The cyst was then imaged using ultrasound and used in conjunction with the clinical images when reviewing the patient at their follow-up clinic appointment with the pediatrician. Figure 5c

\section{Summary}

The transillumination photography techniques and applications described are non- invasive and comply with infection control policies. They are quick and easy to implement with low or no cost implications as no specialist additional equipment is needed. These techniques can be applied to many clinical circumstances, particularly when internal structures need to be imaged and provide additional information to aid diagnosis.

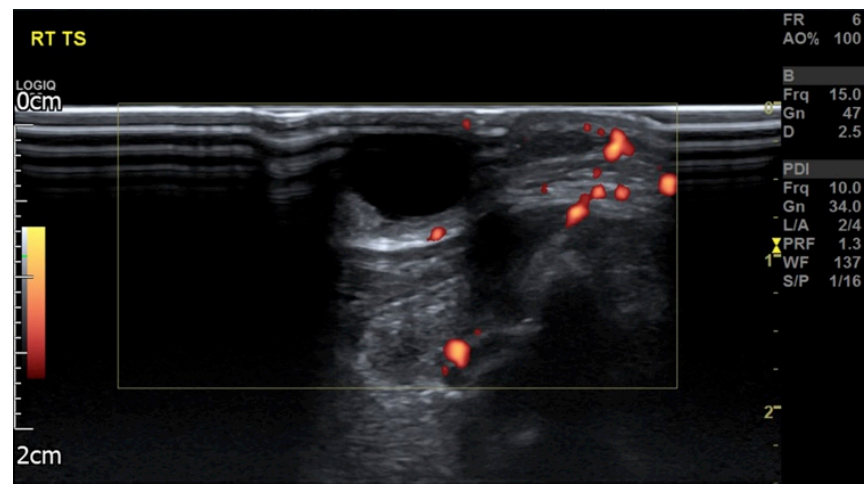

Figure 5c. Ultrasound of cyst.

Being capable of this type of photography sets skilled medical photographers apart from the amateur: being able to communicate well with patients and staff, using professional, efficient techniques applied effectively to give as much visual information as possible to support the patient's clinical record.

\section{Medical Photography - A Personal Perspective}

I love what I do; my mantra is: 'Go back to basics' - using photographic theory knowledge and skills to assess the subject in terms of form, texture, and potential use of image. I apply these factors to the subject, improvising where necessary, aiming to produce images quickly and efficiently that give the impression they have been taken effortlessly in ideal conditions.

\section{A Special Mention}

The Peritoneal Malignancy Institute (PMI) based at Basingstoke and North Hampshire Hospital (BNH) is one of the largest centers in the world treating patients with peritoneal malignancy from around the U.K. and the world.

PMI Basingstoke generously allocated charitable funds to the Medical Photography Department to replace outdated photographic equipment and expand the range of equipment. The funds were donated by the family of Julian Lee, worldrenowned photographer from Hong Kong, who was treated in Basingstoke. His family felt leaving a legacy to support the Medical Photography Service was a fitting tribute to Julian.

Thanks to the charitable funds allocated by the PMI, Hampshire Medical Fund and the Red Cross at Hampshire Hospitals, the Medical Photography Service is able to offer a state-of-the-art professional service throughout Hampshire Hospitals NHS Foundation Trust.

To read more about Julian Lee go to:

https://www.scmp.com/magazines/48hrs/article/1581777/julian -lee-chronicles-his-photographic-career-visionaire-senses 


\section{Acknowledgements}

PMI Basingstoke and North Hampshire Hospital, U.K. https://www.hampshirehospitals.nhs.uk/our-services/azdepartments-and-specialties/peritoneal-malignancy

\section{References}

Chua, T.C., Yan, T.D., Deraco, M., Glehen, O., Moran, B.J., and Sugarbaker, P.H. 2011. Multi institutional experience of diffuse intra abdominal multicystic peritoneal mesothelioma. Br J Surg. 98: 60-64.

Erne, H. C., Gardner, T. R., and Strauch, R. J. 2011. Transillumination of hand tumors: a cadaver study to evaluate accuracy and intraobserver reliability. Hand (New York, N.Y.). 6(4), 390-393.

Gilani S., Mehta A., Garcia-Fadrique A., Rowaiye B., Jenei V., Dayal S., Chandrakumaran K., Carr N., Mohamed F., Cecil T., and Moran B. 2018. Outcomes of cytoreductive surgery with hyperthermic intraperitoneal chemotherapy for peritoneal mesothelioma and predictors of survival. Int J Hyperthermia. 34:5, 578-584.

Goren, A., Laufer, J., Yativ, N., Kuint, J., Ben Ackon, M., Rubinshtein, M., Paret, G., and Augarten, A. 2001. Transillumination of the palm for venipuncture in infants. Pediatr Emerg Care. 17(2), 130-131.

Kaliyadan, F., and Ashique, K. T. 2018. Nail Transillumination Combined with Dermoscopy for Enhancing Diagnosis of Subungual Hematoma. Indian Dermatol Online J. 9(2), 105-106.

Ray, Sidney F. 1999. Scientific photography and applied imaging. Oxford: Boston. Focal Press, 86-87.

\section{Bibliography}

Horisberger, B. and Krompecher, T. 1997. Forensic diaphanoscopy: how to investigate invisible subcutaneous hematomas on living subjects. Int J Leg Med. 110: 73-78.

Kong, X., Cao, Y., Yang, D., and Li, S. 2017. Intraoperative transillumination with water-filling of lumen for localizing lesions in occult small bowel bleeding: Case report. Medicine. 96(5), e6027.

Lara-Capi, C., Cagetti, M. G., Lingström, P., Lai, G., Cocco, F., Simark-Mattsson, C., and Campus, G. 2017. Digital transillumination in caries detection versus radiographic and clinical methods: an in-vivo study. Dentomaxillofac Radiol. 46(4), 20160417.

JBC Vol. 44, No. 1
Reid B. Adams. 2009. Surgical Pitfalls. Enterectomy. Elsevier Inc. ISBN 978-1-4160-2951-9

Russell, N. J., Bassett, P., \& Chang, J. 2018. Low-Cost Bicycle Lights vs. Cold Lights for Visualizing Neonatal Veins. Journal of tropical pediatrics, 64(3), 202-207.

\section{Authors}

Marie Jones, BSc, FIMI, AHCS, PgCHE, FHEA, fCMgr. Marie is the Medical Photography and Illustration Manager for Hampshire Hospitals NHS Foundation Trust, UK. Marie has been a medical photographer for 30 years specialising in photography of cleft lip and palate, and lighting to demonstrate texture and form; these images being used extensively to support the patient's case for NHS funding and for research and teaching. Marie authors and contributes to clinical photography guidelines, professional journal publications and has mentored students and health professionals. As a visiting lecturer, Marie was heavily involved in the development of the clinical photography modules and the teaching and assessment of the BSc (Hons.) Clinical Photography course.

MP054

Hampshire Hospitals NHS Foundation Trust

Basingstoke and North Hampshire Hospital

Aldermaston Road

Basingstoke, United Kingdom

RG24 9NA

Phone: +44 1256313176 Mobile: +44 7785608784

marie.jones13@nhs.net

\section{Faheez Mohamed MD, MBChB, FRCSEd}

Faheez became a Bachelor of Medicine and Surgery at the University of Edinburgh Medical School in 1996. He underwent higher surgical training in the north east of England. He was awarded Doctor of Medicine at the University of Newcastle in 2005 based on his research into intraperitoneal chemotherapy for the treatment of intra-abdominal cancer. $\mathrm{Mr}$. Mohamed has been involved in extensive research on peritoneal surface malignancy under the supervision of Dr. Paul Sugarbaker, Washington DC, USA, and is currently consultant surgeon at the National Commissioning Group Pseudomyxoma Peritonei Centre at Basingstoke and North Hampshire Hospital. Specialist interests: Colorectal cancer; Peritoneal surface malignancy; Academic surgery.

faheez.mohamed@nhs.net 


\section{Licensing}

The author has chosen to license this content under a Creative Commons Attribution, NonCommercial, NoDerivatives License. 4.0 International License

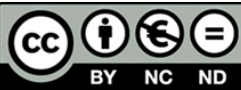

\section{Conflict of Interest Statement}

The Journal of Biocommunication Management Board and Editors believe that transparency in academic research is essential. Our $J B C$ authors are now required to disclose any possible conflict of interest when submitting a paper.

In accordance with the Journal of Biocommunication's editorial policy, no potential conflict of interest has been reported and declared by the author(s). 